- Additional materials are published online only. To view this file please visit the journal online (http://dx.doi.org/ 10.1136/oemed-2011-100469).

1 Institute of Epidemiology II, Helmholtz Zentrum München, German Research Center for Environmental Health, Neuherberg, Germany ${ }^{2}$ Institute of Biometrics and Epidemiology,

Ludwig-Maximilians-University of Munich, Munich, Germany ${ }^{3}$ Department of Environmental Medicine-Lung Biology and Disease Program, University of Rochester School of Medicine and Dentistry, Rochester, New York, USA

${ }^{4}$ Environmental Public Health Division, National Health and Environmental Effects Research Laboratory, Environmental Protection Agency, Durham, North Carolina, USA

${ }^{5}$ Department of Cardiology, University of Ulm, Ulm, Germany ${ }^{6}$ ESC-Environmental Science Center, University of Augsburg, Augsburg, Germany

\section{Correspondence to}

Regina Hampel, Helmholtz Zentrum München, German Research Center for Environmental Health, Institute of Epidemiology II, Ingolstaedter Landstr. 1, 85764 Neuherberg. Germany; regina.hampel@ helmholtz-muenchen.de

CLS and RH shared first authorship.

Accepted 14 April 2012 Published Online First 25 July 2012

ORIGINAL ARTICLE

\title{
Short-term effects of air temperature on blood markers of coagulation and inflammation in potentially susceptible individuals
}

\author{
Claudia Luise Schäuble, ${ }^{1}$ Regina Hampel, ${ }^{1}$ Susanne Breitner, ${ }^{1,2}$ Regina Rückerl, ${ }^{1}$ \\ Richard Phipps, ${ }^{3}$ David Diaz-Sanchez, ${ }^{4}$ Robert B Devlin, ${ }^{4}$ Jacqueline D Carter, ${ }^{4}$ \\ Joleen Soukup, ${ }^{4}$ Robert Silbajoris, ${ }^{4}$ Lisa Dailey, ${ }^{4}$ Wolfgang Koenig, ${ }^{5}$ Josef Cyrys, ${ }^{1,6}$ \\ Uta Geruschkat, ${ }^{1}$ Petra Belcredi, ${ }^{1}$ Ute Kraus, ${ }^{1,2}$ Annette Peters, ${ }^{1}$ \\ Alexandra E Schneider ${ }^{1}$
}

\section{ABSTRACT}

Objectives Changes in air temperature are associated with an increase in cardiovascular events, but the role of procoagulant and proinflammatory blood markers is still poorly understood. The authors investigated the association between air temperature and fibrinogen, plasminogen activator inhibitor type 1, interleukin-6 and high-sensitivity $C$ reactive protein in two potentially susceptible groups.

Methods This prospective panel study was conducted between March 2007 and December 2008 in Augsburg, Germany. The study population comprised 187 participants with type 2 diabetes mellitus or impaired glucose tolerance and 87 participants with genetic polymorphisms on the detoxification and inflammation pathways. Overall, 1766 repeated blood measurements were collected. Hourly meteorology data were available from a central measurement site. The association between temperature and blood markers was analysed with additive mixed models.

Results For type 2 diabetes mellitus and impaired glucose tolerance participants, the authors observed immediate, lagged and cumulative increases in fibrinogen (range of percentage changes in geometric mean: $0.6 \%-0.8 \%$ ) and plasminogen activator inhibitor type 1 $(6.0 \%-10.1 \%)$ in association with a $5^{\circ} \mathrm{C}$ temperature decrement. Participants with a body mass index above $30 \mathrm{~kg} / \mathrm{m}^{2}$ as well as females showed particularly strong fibrinogen effects. In participants with the special genetic background, $5^{\circ} \mathrm{C}$ decreases in the 5-day average of temperature led to a change of $8.0 \%(95 \% \mathrm{Cl} 0.5 \%$ to $16.2 \%)$ in interleukin-6 and of $-8.4 \%(95 \% \mathrm{Cl}-15.8 \%$ to $-0.3 \%$ ) in high-sensitivity $C$ reactive protein, the latter driven by physically active individuals.

Conclusions The authors observed different temperature effects on blood markers in two potentially susceptible groups probably indicating varying underlying biological mechanisms. This study results might provide a link between temperature and cardiovascular events.

\section{INTRODUCTION}

Increases as well as decreases in air temperature have been shown to be associated with higher morbidity $^{12}$ and mortality ${ }^{3-5}$ due to cardiovascular disease (CVD). Various procoagulant and proin-

\section{What this paper adds}

- This paper contributes to the understanding of associations between air temperature and cardiovascular events, for which no clear link has been established yet, by looking at intermediate markers associated with CVD in relation to changes in temperature.

- Only few studies have examined associations between temperature and $C$ reactive protein, fibrinogen and IL-6, whereas so far no study investigated temperature effects on plasminogen activator inhibitor 1.

- Potentially susceptible individuals, in particular subjects with type 2 diabetes or IGT and those with a genetic predisposition on the detoxification or inflammation pathways, were selected for this study.

- We observed significant changes in blood markers in association with temperature decreases which differed between the two groups and which were modified by sex, BMI and physical activity.

- Our study might help to identify susceptible individuals which should take precautions in special weather conditions and might help to explain the differences in susceptibility to air temperature seen in other studies.

flammatory blood markers are predictors for acute or chronic CVD. ${ }^{6-9}$ Especially fibrinogen and $\mathrm{C}$ reactive protein $(\mathrm{CRP})$ seem to be predictors that are independent of traditional risk factors such as increased waist circumference, low physical activity, smoking and diabetes. ${ }^{6}$

Some blood markers show a seasonal variation. While many studies ${ }^{10-12}$ observed higher fibrinogen levels in winter, a study of Rudez et $a l^{13}$ observed higher levels in summer. It has also been shown that CRP levels are higher in winter and spring. ${ }^{14}$ Furthermore, Kanikowska et al ${ }^{15}$ detected significantly higher blood levels of interleukin-6 (IL-6) in winter and summer compared with spring and autumn. 
Physiological mechanisms explaining the potential association between temperature changes and cardiovascular events are still poorly understood. Previous studies investigating the temperature effect of controlled exposure to cold water or cold air showed elevated levels of risk factors for CVD-among them, an increase of plasminogen activator inhibitor type 1 (PAI-1), ${ }^{16}$ fibrinogen, ${ }^{17}$ red blood cells, blood viscosity ${ }^{18}$ and blood pressure. ${ }^{19}$ In a European study with repeated measurements of fibrinogen, IL-6 and $\mathrm{CRP}$ in myocardial infarction (MI) survivors, temperature decreases 3 days before blood withdrawal were associated with an increase in fibrinogen. ${ }^{20}$ Moreover, elevated levels of IL- 6 and CRP in association with decreases in the 5-day average of temperature were detected in the same study. A study from the Boston area in elderly men showed temperature effects only for CRP but not for IL-6. ${ }^{21}$ CRP increased due to temperature decreases up to 1 day before blood withdrawal and decreases in the 4-week average of temperature. ${ }^{21}$ Furthermore, Hampel et al ${ }^{22}$ found decreases in $\mathrm{CRP}$ and fibrinogen in association with a $10^{\circ} \mathrm{C}$ temperature decrease in patients with coronary heart disease (CHD).

Elderly and individuals with diabetes ${ }^{2} 23$ are assumed to be more susceptible to temperature changes than others. Accordingly, Wolf et $a l^{2}$ observed an association between temperature drops and MI only in individuals above 55 years of age, whereas younger subjects did not react to temperature decreases. Schwartz ${ }^{23}$ detected a higher risk of mortality on hot days in individuals with diabetes compared with other individuals.

In this study, we investigated immediate, lagged and cumulative effects of ambient air temperature on repeatedly measured blood markers of coagulation and inflammation in a moderate climate. We assessed the blood markers fibrinogen, PAI-1, IL-6 and high-sensitivity $\mathrm{C}$ reactive protein (hsCRP) in a group of individuals with type 2 diabetes mellitus (T2D) or impaired glucose tolerance (IGT), a prediabetic state, and in individuals with genetic polymorphisms on the detoxification and inflammation pathways but without T2D or IGT. Our hypothesis was to observe increases in blood markers in association with temperature drops in participants with T2D or IGT as well as in participants with the specific genetic background.

\section{MATERIALS AND METHODS}

\section{Study design and study population}

A prospective panel study was conducted between 19 March 2007 and 17 December 2008 in Augsburg, Germany, as part of the University of Rochester Particulate Matter Center investigations. Individuals with T2D or IGT and healthy individuals with a special genetic background were recruited from the KORA (Cooperative Health Research in the Region of Augsburg) F4 cohort that was conducted between 2006 and 2008. ${ }^{25} 26$ The participants were living in Augsburg or in the two adjacent counties. T2D was diagnosed either by a physician or a typical medication use (ATC-Code A10). Remaining participants had an oral glucose tolerance test (OGTT). Participants with a fasting glucose level $>125 \mathrm{mg} / \mathrm{dl}$ or a $2 \mathrm{~h}$ OGTT glucose level $\geq 200 \mathrm{mg} /$ $\mathrm{dl}$ were also specified as having T2D. IGT was specified as $2 \mathrm{~h}$ OGTT glucose levels $\geq 140 \mathrm{mg} / \mathrm{dl}$ but $<200 \mathrm{mg} / \mathrm{dl}$. The special genetic background was defined as having the null polymorphism for GSTM1 and either two major alleles of the single-nucleotide polymorphism (SNP) rs1205 located in the CRP gene or at least one minor allele of the SNP rs1800790 located in the fibrinogen gene FGB. GSTM1 is assumed to be involved in the protection against oxidative stress and hence in inflammatory processes. ${ }^{27}$ The two latter SNPs have been shown to have an influence on CRP and fibrinogen levels, respectively. ${ }^{28-30}$ The individuals were invited to up to seven examinations scheduled every 4-6 weeks on the same day of the week and the same time of the day. At the first examination, data on health status, medication use as well as disease and smoking history were collected. For a subgroup of the participants also an electrocardiogram was recorded. Therefore, diseases or participant characteristics with a potential influence on blood markers or cardiac function led to exclusion of participants or single examinations. In detail, exclusion criteria were current smoking or being an ex-smoker for $<12$ months; intake of platelet aggregation inhibitors except for acetylsalicylic acid (ASA); a MI and/or interventional procedure (percutaneous transluminal coronary angioplasty, bypass surgery) $<6$ months before the start of the study and chronic inflammatory diseases such as Crohn's disease, colitis ulcerosa and rheumatoid arthritis. Single blood samples of the participants were excluded if the participant had a cold/influenza or a surgery or dental surgeon intervention or an acute infection of the urological/gastro-intestinal/respiratory tract during the 3 days prior the examination. Furthermore, if a participant was not in the study area before the blood withdrawal, the respective blood sample was excluded from analyses. All methods used in the study were conducted according to Standard Operating Procedures. The study protocol was accredited by the Ethics Commission of the Bavarian Chamber of Physicians (Bayerische Landesaerztekammer) and all participants gave written informed consent.

\section{Clinical measurements}

At each visit, a venous blood withdrawal was conducted. For the analysis of blood markers, ethylenediaminetetraacetic acid and citrate samples were drawn (Becton Dickinson, Franklin Lakes, New Jersey, USA) at each visit. Samples were centrifuged at $4^{\circ} \mathrm{C}$ and aliquots were immediately stored at $-80^{\circ} \mathrm{C}$ until shipment on dry ice to the collaborating laboratories. We investigated the coagulation markers fibrinogen and PAI-1 as well as the inflammatory markers IL-6 and hsCRP. Fibrinogen was analysed by immunoephelometry (Dade Behring, Marburg, Germany). PAI-1 was determined by a home-made assay using the mesoscale platform, and determination of IL- 6 was accomplished using an IL-6 Ultrasensitive Kit (Meso Scale diagnostics, Gaithersburg, Maryland, USA). HsCRP was analysed by a Meso Scale Discovery Kit (Meso Scale diagnostics). Only individuals with at least two valid blood samples were considered for the analysis.

\section{Meteorology and air pollution data}

Hourly means of air temperature, relative humidity, barometric pressure and air pollutants such as ultrafine particles (UFP; particle number count, with a size range of $0.01-0.1 \mu \mathrm{m}$ in aerodynamic diameter), $\mathrm{PM}_{2.5}$ (particulate matter (mass) with a size range of $<2.5 \mu \mathrm{m}$ in aerodynamic diameter) and $\mathrm{PM}_{10}$ (particulate matter (mass) with a size range of $<10 \mu \mathrm{m}$ in aerodynamic diameter) were measured at a central measurement site in Augsburg throughout the study period. ${ }^{31} 32$ Ozone was measured as hourly means at a monitoring site operated by the Bavarian Environment Agency (LfU). Missing values of meteorology and air pollution data were not replaced since $<1 \%$ of $24 \mathrm{~h}$ averages were missing (meteorological variables, ozone, $\mathrm{PM}_{2.5}, \mathrm{PM}_{10}$ ) or no parallel measurement with other devices (UFP) was available. For each participant and visit, we calculated individual $24 \mathrm{~h}$ averages of each meteorological variable preceding the blood withdrawal (lag 0: 0-23 h) for up to 4 days (lag 1: 24-47 h, lag 2: 48-71 h, lag 3: 72-95 h, lag 4: 96-119 h) if more than two-thirds of the hourly measurement were available. In addition, we calculated the mean of these 5 days (5day average). For a more detailed description of the meteorological and air pollution data, see supplemental material. 


\section{Statistical analysis}

Data analysis was performed separately for two groups consisting of individuals with T2D or IGT and participants with a special genetic background, respectively. Medians of patientspecific Spearman correlation coefficients were calculated between all blood markers. Patient characteristics were compared between the two groups using a Student t test for continuous variables and a $\chi^{2}$ test for categorical variables. Fisher's exact test was used if one category contained less than five observations. Mixed models with a random participant effect and including dummy variables for the group effect were calculated in order to compare the blood markers between the groups. Because the halflives of the blood markers were shorter than the intervals between the visits, we assumed a compound symmetry structure for the covariance matrix. The $p$ value of the group effect indicates whether the blood markers differ significantly between the two groups. In order to analyse air temperature effects on blood markers, we used additive mixed models with a random participant effect and a compound symmetry covariance structure. Data on blood markers were log-transformed to obtain normally distributed residuals. A confounder selection with longterm time trend, relative humidity, barometric pressure and day of the week was conducted for each blood marker separately. Model selection was done by minimising the Akaike's Information criterion. Long-term time trend and relative humidity with the same lag as the analysed temperature lag were forced into the model. Barometric pressure and weekday were only included in case of model fit improvement. Continuous confounders were included linearly or smoothly as penalised spline (P-spline) to allow for non-linear exposure-response functions. ${ }^{33}$ If trend or meteorological variables such as relative humidity and barometric pressure were included as a P-spline, we checked whether a polynomial led to a better model fit. Observations with obviously extremely large residuals in the confounder model were excluded from further analysis. After assessing the confounder models, single temperature lags were included either linearly or as a P-spline to the confounder model. All analyses were performed using SAS V.9.2 (SAS Institute Inc.).

\section{Effect modification and sensitivity analysis}

Season (winter: October to March vs summer: April to September), gender (female vs male), body mass index (BMI) $\left(<30 \mathrm{~kg} / \mathrm{m}^{2}\right.$ vs $\left.\geq 30 \mathrm{~kg} / \mathrm{m}^{2}\right)$, age ( $<60$ vs $\geq 60$ years), physical activity (not active vs $\geq 1 \mathrm{~h} /$ week), intake of statins (yes vs no) and intake of ASA (yes vs no) were considered as potential temperature effect modifiers by including a binary interaction term in the model. In participants with T2D or IGT, we additionally calculated an interaction analysis with a variable indicating whether the participant also had the specific genetic background.

Moreover, we studied the robustness of the temperature effects by performing several sensitivity analyses. We additionally adjusted our models for UFP, $\mathrm{PM}_{2.5}, \mathrm{PM}_{10}$ or ozone with the same lag as temperature. Furthermore, we excluded blood marker measurements below the 5th or above the 95th percentile to avoid overestimation of effects induced by extreme blood marker values.

\section{RESULTS}

\section{Study population and clinical measurements}

Overall, 276 participants were examined and 1799 blood samples were drawn. One participant with seven blood withdrawals was excluded because of Loefgren syndrome. One participant was excluded because only one valid blood sample was available.
Twenty-three blood samples were excluded from analysis because of an acute disease or surgery within 3 days before the clinical examination. One participant had MI between his fifth and sixth visits; therefore, the sixth blood sample was excluded. One blood sample was not used for analysis because the participant spent the whole morning before the blood draw outside the study area. Therefore, temperature measurements from the central monitoring site in Augsburg preceding the examination cannot be assigned to this visit. Finally, 1766 valid blood samples of 274 participants were available for analysis. Two hundred and twenty-two participants (80.7\%) completed all seven visits. The study population comprised 187 (68\%) participants with T2D or IGT with 1197 blood samples and 87 (32\%) participants with the specific genetic background with 569 blood samples. Clinical characteristics of the participants are described in table 1. Participants with T2D or IGT were significantly older, more overweight and hypertensive, less physically active and took more statins and acetylsalicylic acid than those with the special genetic background. Six hundred and seventynine (57\%) and 273 (48\%) examinations were performed in summer in participants with T2D or IGT and a genetic predisposition, respectively. Fibrinogen, PAI-1, IL- 6 and hsCRP were higher in participants with T2D or IGT. According to the medians of the patient-specific correlation coefficients, the blood markers showed only a low correlation $(|r|<0.4)$ (table 2). For both study groups, all blood markers were slightly higher in winter compared with summer except for IL-6 in participants with the special genetic background (data not shown).

\section{Meteorological data}

During the study period (645 days), $24 \mathrm{~h}$ averages were $10.9^{\circ} \mathrm{C}$ (SD 7.3) for temperature, 77\% (SD 12.6) for relative humidity and $961.3 \mathrm{hPa}$ (SD 7.6) for barometric pressure. Meteorological variables were only weakly correlated $(|\mathrm{r}|<0.5)$, whereas UFP with $\mathrm{PM}_{2.5} / \mathrm{PM}_{10}$ showed a moderate $(\mathrm{r}<0.6)$ and $\mathrm{PM}_{2.5}$ and $\mathrm{PM}_{10}$ showed a strong correlation $(\mathrm{r}=0.93)$. Ozone was negatively correlated with meteorological variables and other air pollutants ( $\mathrm{r}=-0.55$ to -0.36 ) (see supplemental table 1 ).

\section{Association of air temperature and blood markers}

In a first step, we added temperature as a P-spline to the models in order to allow for a non-linear temperature-response relationship. There was no evidence in either group for a deviation from linearity for the relationship between temperature and blood markers (supplemental figure 1). Therefore, temperature lags were included linearly in the final models. The selected confounder models for each blood marker are summarised in supplemental table 2. Linear temperature effects on the blood markers are presented as per cent change from the geometric outcome mean per $5^{\circ} \mathrm{C}$ decrease in temperature together with $95 \%$ CIs (figure 1). We observed immediate, lagged and cumulative increases in fibrinogen and PAI-1 in association with a drop in temperature in participants with T2D or IGT. A decrease in the 5-day average of temperature led to the strongest increase in fibrinogen $(0.8 \%(95 \%$ CI $0.3 \%$ to $1.3 \%))$ and PAI-1 $(10.1 \%(95 \%$ CI $6.4 \%$ to $13.8 \%)$ ), respectively. Participants with the genetic predisposition showed no temperature effects in both blood markers. We observed effects of a temperature decrease on IL-6 with a lag of one $(6.2 \%(95 \%$ CI $0.4 \%$ to $12.4 \%))$ up to 4 days $(5.9 \%(95 \%$ CI $0.3 \%$ to $11.9 \%))$ as well as for the 5 -day average $(8.0 \%(95 \%$ CI $0.5 \%$ to $16.2 \%))$ only in participants with the special genetic background. In the same panel, hsCRP decreased for lag $0(-9.5 \%(95 \% \mathrm{CI}-16.6 \%$ to $-1.8 \%))$, lag $1(-8.0 \%(95 \%$ CI $-15.0 \%$ to $-0.4 \%)$ ), lag $2(-7.7 \%(95 \%$ CI $-14.5 \%$ to 
Table 1 Description of clinical characteristics and levels of blood markers in study participants

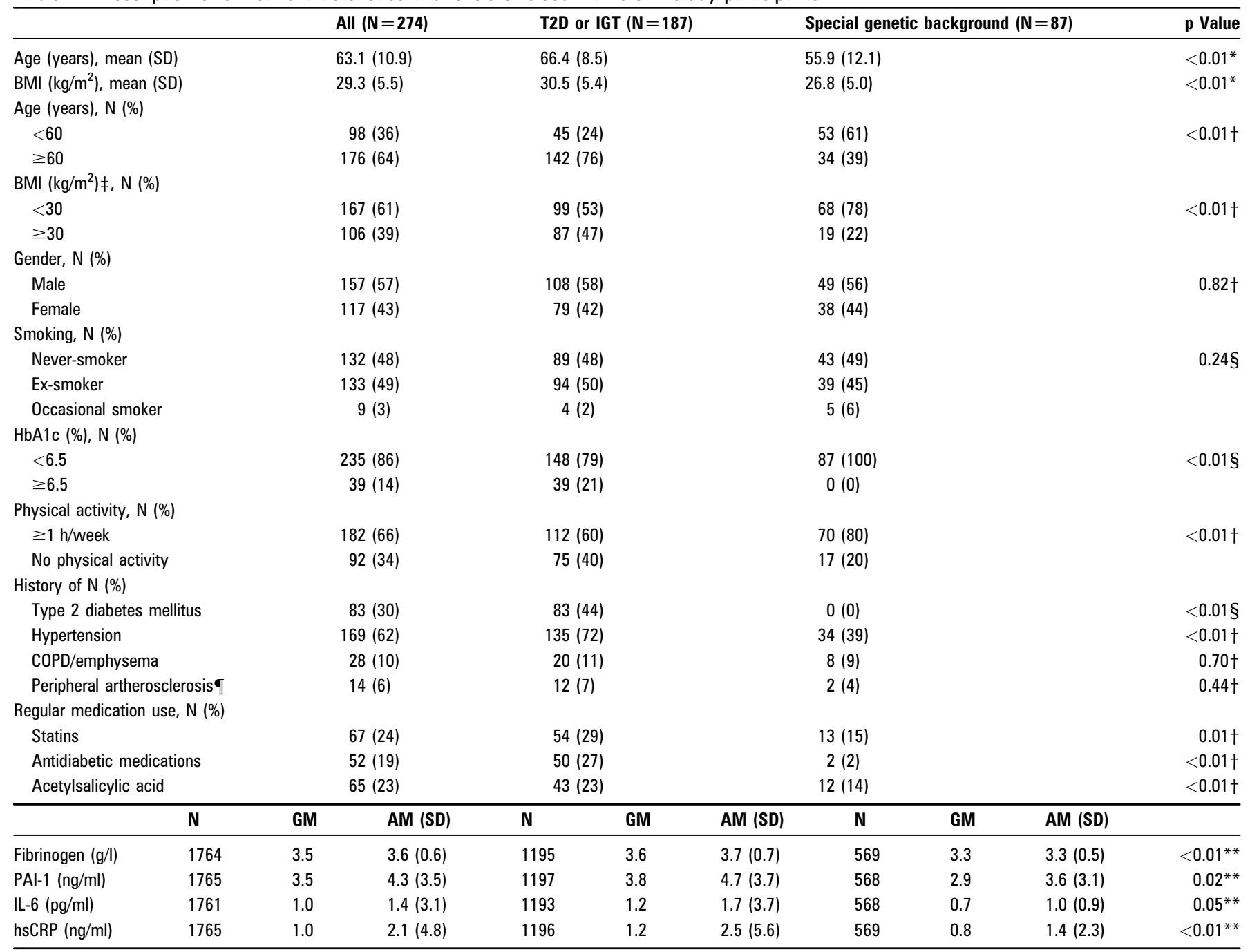

*Student's t test.
$\dagger \chi^{2}$ test.
†One participant without BMI measurement.
§Fisher's exact test.
\Forty-nine participants without data.
${ }^{* *}$ p Value of fixed group effect in mixed effects model.
AM, arithmetic mean; BMl, body mass index; COPD, chronic obstructive pulmonary disease; GM, geometric mean; HbA1c, glycosylated haemoglobin; hsCRP, high-sensitivity C reactive protein;
IGT, impaired glucose tolerance; IL-6, interleukin-6; PAl-1, plasminogen activator inhibitor type 1; T2D, type 2 diabetes mellitus.

$-0.4 \%))$, lag $3(-6.5 \%(95 \% \mathrm{CI}-13.1 \%$ to $0.6 \%))$ and for the 5 day average $(-8.4 \%(95 \% \mathrm{CI}-15.8 \%$ to $-0.3 \%))$ in association with a temperature decrement, whereas we found no temperature effects on hsCRP in participants with T2D or IGT.

\section{Effect modifications}

Selected temperature effect modifications in participants with T2D or IGT are shown in figure 2. Female participants showed an increase in fibrinogen of about $1 \%$ in all lags in association

Table 2 Medians of the patient-specific Spearman correlation coefficients of blood markers

\begin{tabular}{llll}
\hline & Fibrinogen & PAI-1 & IL-6 \\
\hline Fibrinogen & & & \\
PAI-1 & 0.00 & 0.00 & \\
IL-6 & 0.21 & 0.07 & 0.18 \\
hsCRP & 0.39 & & \\
\hline \multicolumn{4}{l}{ hsCRP, high-sensitivity C reactive protein; IL-6, interleukin 6; PAl-1, } \\
plasminogen activator inhibitor type 1.
\end{tabular}

with temperature decreases, while we observed no temperature effects in men. Furthermore, we observed an increase in fibrinogen of about $1 \%$ in all lags only in participants with a BMI $\geq 30 \mathrm{~kg} / \mathrm{m}^{2}$. Participants taking statins tended to show slightly stronger temperature effects on PAI-1 and hsCRP (figure 2). We observed a stronger decrease in hsCRP during winter compared with summer in association with a temperature drop in participants with the genetic predisposition (figure 3). In the same panel, the decrease in hsCRP seemed to be more pronounced in physically activity individuals, whereas individuals without physical activity tended to slightly elevated fibrinogen levels in association with temperature decreases (figure 3). No further temperature effect modifications on any blood marker were detected neither in participants with metabolic disorders nor in those with the special genetic background. Forty-six participants with T2D or IGT also had the specific genetic background. However, temperature effects were similar in participants with and without the genetic predisposition (data not shown). 

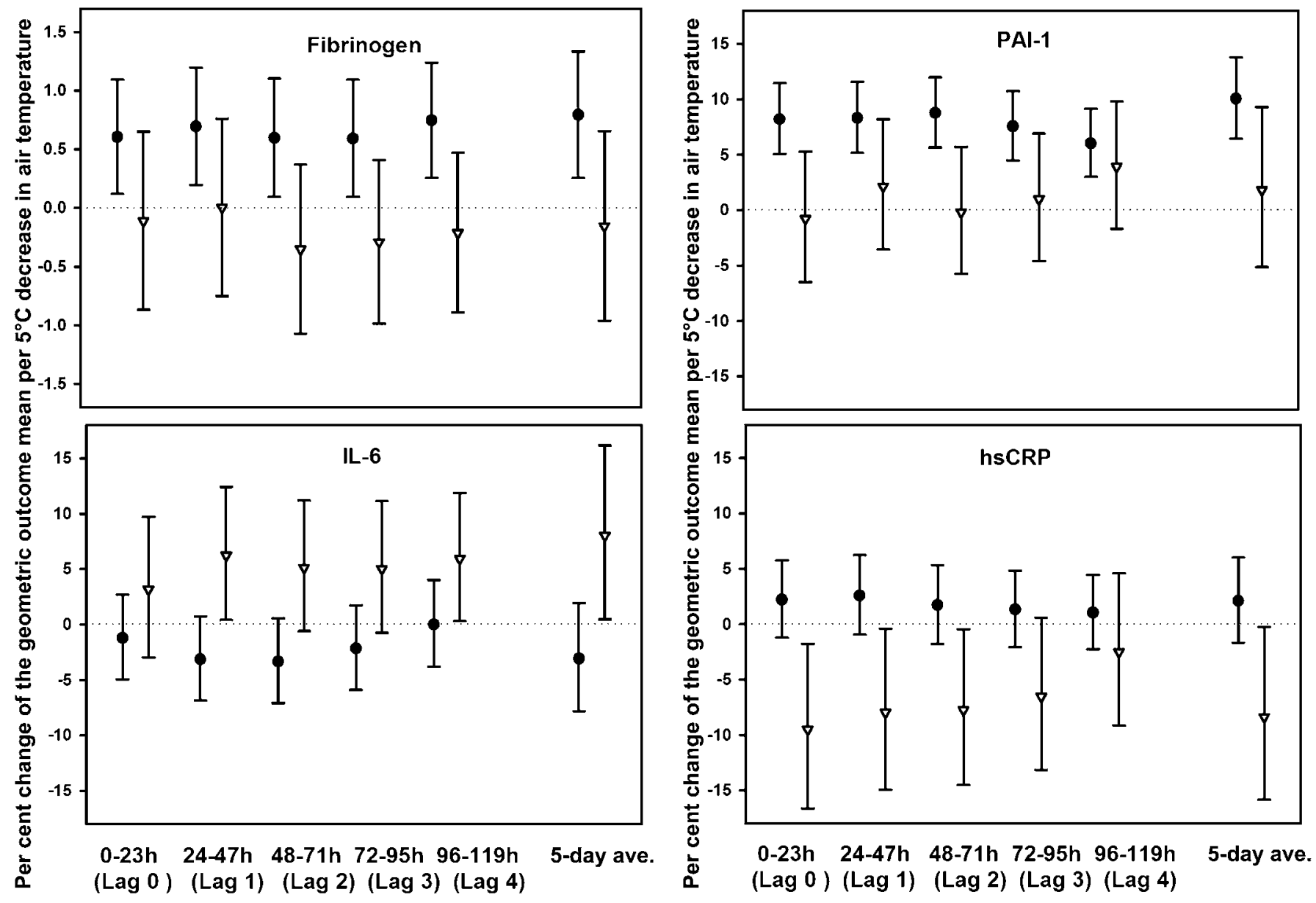

\section{- T2D or IGT \\ $\nabla \quad$ Special genetic background}

Figure 1 Air temperature effects on fibrinogen, plasminogen activator inhibitor type 1 (PAI-1), interleukin-6 (IL-6) and high-sensitivity C reactive protein (hsCRP).

\section{Sensitivity analyses}

Adjusting the models for UFP, $\mathrm{PM}_{2.5}$ and $\mathrm{PM}_{10}$ did not change temperature effects on fibrinogen, PAI-1, IL-6 and hsCRP. However, temperature effects on IL- 6 with a lag of 1 and 4 days became non-significant when adjusting for ozone in participants with the special genetic background (supplemental table 1). Excluding blood samples with values below the 5 th and above the 95th percentile led to non-significant temperature effects (lags $0-3$ ) on fibrinogen in participants with T2D or IGT (supplemental table 3).

\section{DISCUSSION}

\section{Summary}

In this study, we investigated short-term temperature effects in two potentially susceptible groups. Only participants with T2D or IGT showed immediate, lagged and cumulative increases in fibrinogen and PAI-1 levels in association with a decrease of $5^{\circ} \mathrm{C}$ in air temperature. In this group, temperature effects on fibrinogen were stronger in women and in obese participants. We did not find temperature effects on IL- 6 and hsCRP in this group. However, a drop of $5^{\circ} \mathrm{C}$ in temperature led to elevated IL- 6 levels and a decrease in hsCRP in participants with a special genetic background. This association was more pronounced in physically active individuals.

\section{Air temperature and blood markers}

Levels of blood markers seem to increase mostly during the winter. ${ }^{10} 1415$ Hence, changes in blood markers due to temperature changes might precede cardiovascular events. However, knowledge about the effects of temperature on blood markers is still limited. We observed inverse temperature effects on fibrinogen and PAI-1 only in individuals with T2D or IGT. In accordance to our results, Schneider et al ${ }^{20}$ and Hampel et al ${ }^{22}$ found elevated fibrinogen levels in association with temperature decrements in MI survivors and in participants with pulmonary disease. A decrease of $10^{\circ} \mathrm{C}$ in the 5 -day average of temperature led to a $5.5 \%$ (95\% CI $1.3 \%$ to $9.7 \%$ ) increase in fibrinogen in pulmonary disease participants, ${ }^{22}$ which is stronger compared with our observed $1.6 \%$ (95\% CI $0.5 \%$ to $2.7 \%$ ) increase in fibrinogen for the same temperature decline. Schneider et al ${ }^{20}$ detected the strongest increase of fibrinogen of about 1.3\% $195 \%$ CI $0.2 \%$ to $2.4 \%$ ) for lag 3 , which is very similar compared with our observed increase of $1.2 \%$ (95\% CI $0.2 \%$ to $2.2 \%$ ) for a temperature decrement of $10^{\circ} \mathrm{C}$. In our participants with $\mathrm{T} 2 \mathrm{D}$ or IGT, a decrease in temperature led to elevated levels of PAI-1 that is among others synthesised in adipocytes and has a prothrombotic function. ${ }^{34}$ In our study, participants with T2D or IGT were more overweight and showed higher PAI-1 levels than those with the special genetic background. Adipose tissue 


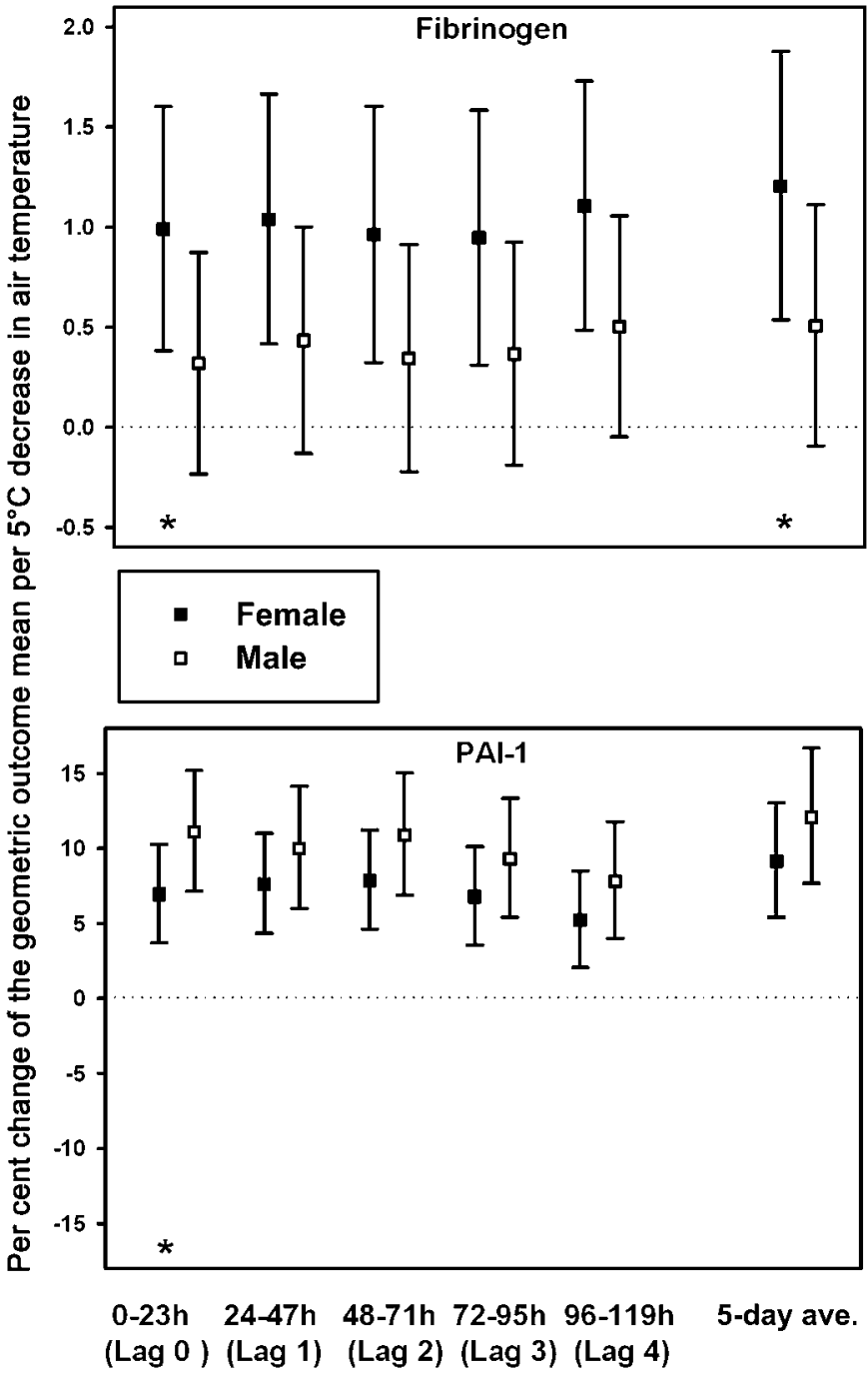

- No statin intake

- Statin intake
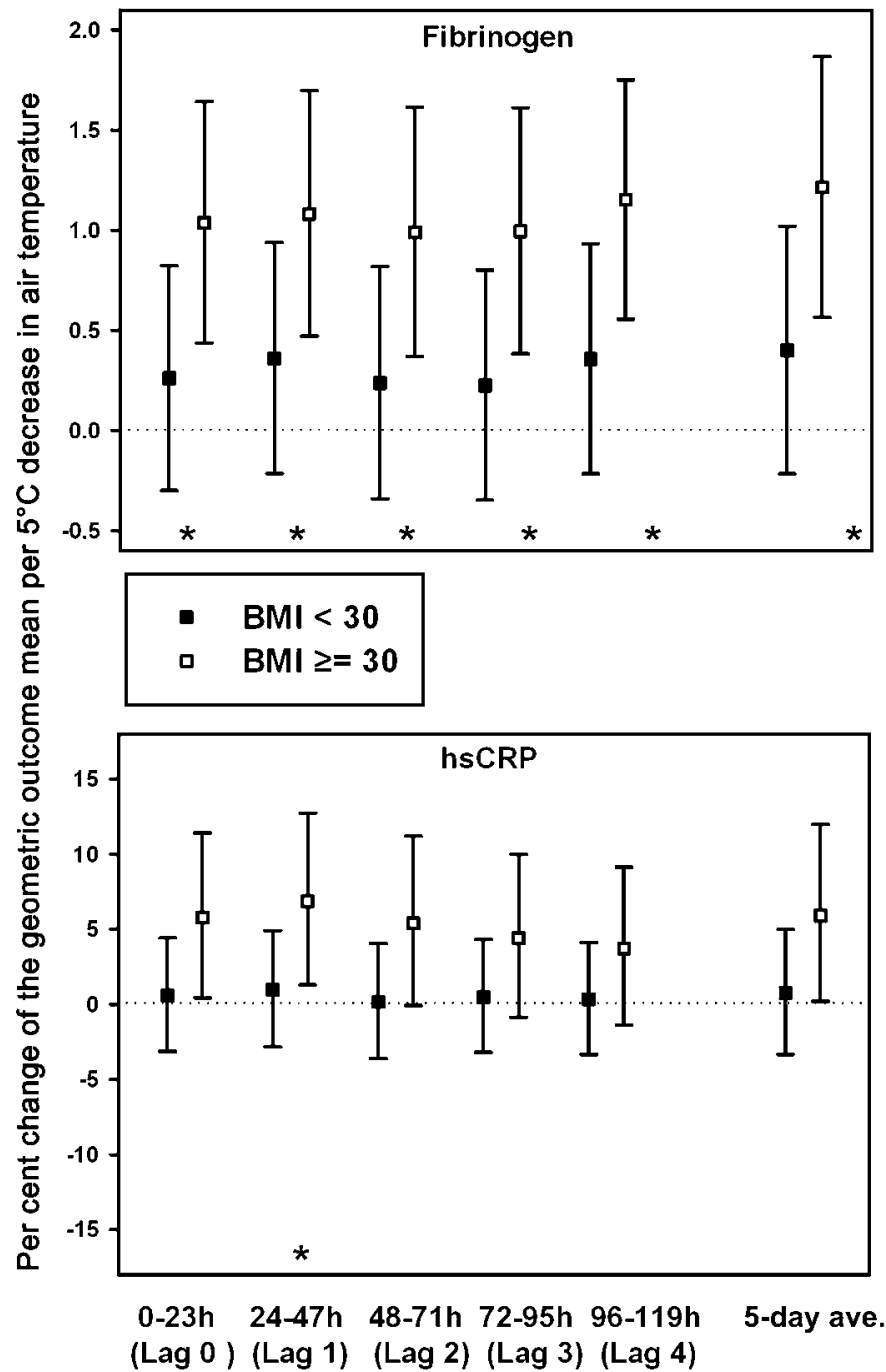

\section{- No statin intake a Statin intake}

Figure 2 Air temperature effects on fibrinogen, plasminogen activator inhibitor type 1 (PAl-1), interleukin 6 (IL-6) and high-sensitivity C reactive protein (CRP) in participants with type 2 diabetes mellitus (TD2) or impaired glucose tolerance (IGT) modified by gender, body mass index (BMI) and intake of statins.

and T2D are both associated with metabolic disorders and a lowgrade systemic chronic inflammation, which increases the risk of CVD. ${ }^{34}$ We speculate that an additional increase in PAI-1 due to a temperature decrease might partly explain the increased diabetes-related mortality in cold months. ${ }^{35}$ Our findings suggest a stimulation of the IL-6 production in association with a decrease in temperature in participants with the special genetic background. Elevated blood levels of IL- 6 induce the production of acute-phase proteins such as hsCRP in the liver. In contrast to our initial hypothesis and other studies, ${ }^{20}{ }^{21}$ we found decreases in hsCRP and no change in fibrinogen in association with a temperature decrease for participants with the potential genetic predisposition. However, a previous study by Hampel et $a^{22}$ also detected a short-term decrease in CRP associated with a drop in temperature. We can only speculate that our findings are a consequence of the special genetic background specified by the null polymorphism of GSTM1 and either two major alleles of the SNP rs1205 located in the CRP gene or at least one minor allele of the SNP rs1800790 located in the fibrinogen gene FGB.
Studies found higher ${ }^{28}$ and lower ${ }^{30}$ hsCRP levels in individuals with at least one minor allele of the rs1205. Having at least one minor allele of the rs1800790 seems to be associated with higher fibrinogen levels. ${ }^{29}$ Tang et $a l^{36}$ reported higher hsCRP and fibrinogen levels in patients with the null polymorphism of GSTM1 compared with patients with the wild type of GSTM1. However, elevated levels of fibrinogen and hsCRP are also common in individuals with T2D. ${ }^{37} 38$ In line with these findings, participants with T2D or IGT in our data had higher fibrinogen and hsCRP levels than those with the special genetic background. We therefore assume that a metabolic disorder like T2D or IGT might have stronger influence on levels of blood markers of fibrinogen or hsCRP and on temperature sensitivity than the defined genetic background.

\section{Potential mechanisms}

The physiological mechanisms leading from temperature changes to cardiovascular events are still not clearly established. Exposure to cold can have various effects like an increase of 


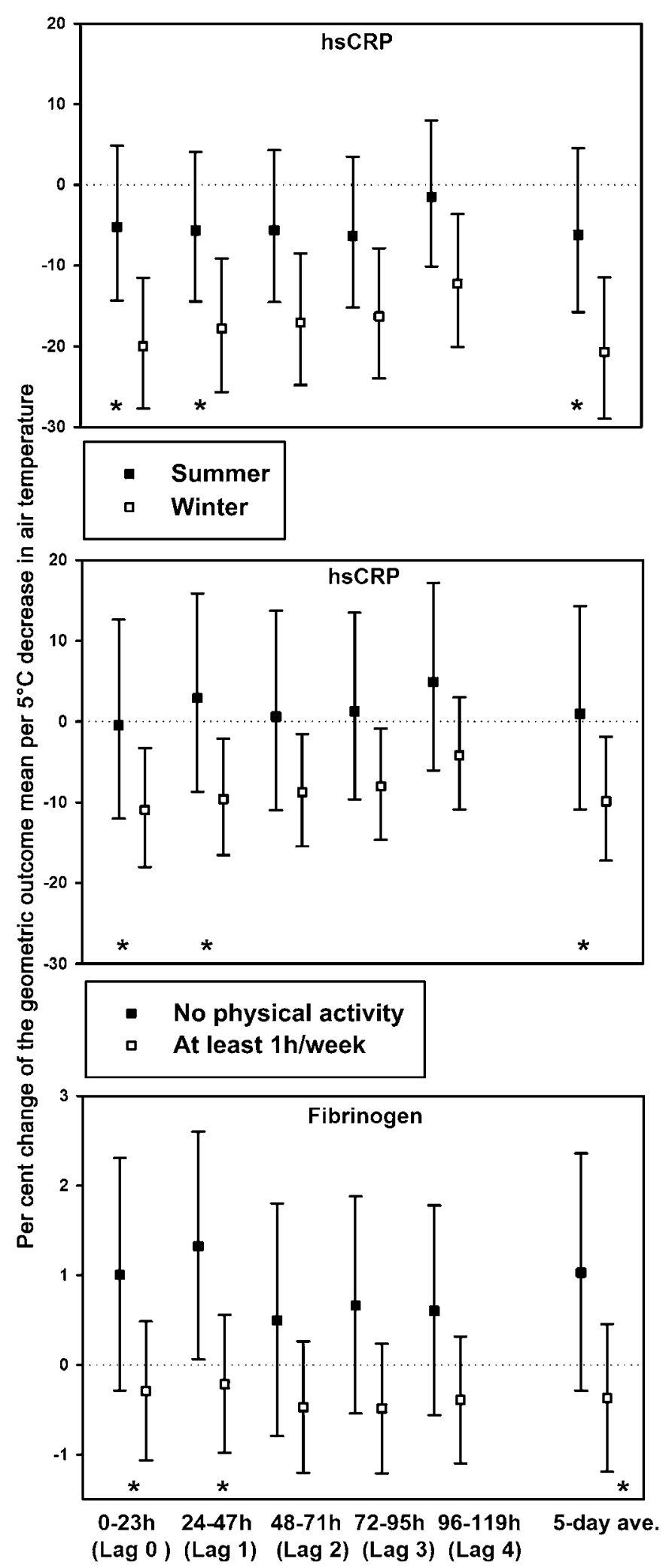

- No physical activity

a At least $1 \mathrm{~h} /$ week

Figure 3 Air temperature effects on fibrinogen and high-sensitivity $C$ reactive protein (CRP) in participants with special genetic background modified by season and physical activity. blood pressure and heart rate ${ }^{18} 1939$ as well as changes in blood markers. Elderly individuals seem to be more susceptible to cold temperature compared with younger individuals because they showed a higher increase of blood pressure after superficial skin cooling $^{19}$ and higher longitudinal changes in blood pressure regarding seasonal temperature changes. ${ }^{39}$ There is also evidence for stronger temperature effects in individuals with T2D. Accordingly, Schwartz ${ }^{23}$ reported a higher mortality risk on hot days in individuals with diabetes than in those without diabetes. Since procoagulatory and proinflammatory blood markers are predictors for $\mathrm{CVD},{ }^{7-9}$ temperature effects on blood markers in subjects with diabetes are also of interest as this maybe one possible biological pathway linking temperature changes and mortality in individuals with T2D. Accordingly, Hampel et al ${ }^{22}$ observed stronger changes in blood markers of coagulation in association with temperature decreases in participants with a CHD and T2D than in those with a CHD but without T2D. Earlier studies suggested that controlled exposure to cold air or water led to an increase in blood markers like fibrinogen ${ }^{16}$ and PAI-1. ${ }^{17}$ Furthermore, it has been shown that a short-term decrease in temperature was associated with increased levels of fibrinogen, IL- 6 and CRP. ${ }^{20}$ Fibrinogen, ${ }^{6}$ IL- $6^{7}$ and CRP ${ }^{6-8}$ are established predictors of cardiovascular risk and IL-6 and CRP seem to be more associated with fatal than non-fatal cardiovascular events. ${ }^{9}$ Several blood markers like fibrinogen, IL-6 and CRP showed seasonal variations with increases mostly in the cold season ${ }^{10-121415}$ and might therefore be one possible link to the peak of cardiovascular events in winter. However, in our study, temperature effects were inconsistent between participants with T2D or IGT and participants with a special genetic background. Participants with T2D or IGT were older, more often overweight, more strongly medicated whereupon participants with T2D took more statins and antidiabetic medications compared with participants with IGT and had a worse overall health than those with the special genetic background. A combination of these factors might have influenced the susceptibility to temperature.

\section{Air temperature effect modifications}

In participants with T2D or IGT, we found increases in fibrinogen in association with a temperature decrement in women only possibly indicating a gender dimorphism in thermoregulation. In accordance with our findings, cold-related cardiovascular symptoms like chest pain or arrhythmia seem to be more common in women than men. ${ }^{40}$ Furthermore, Schwartz ${ }^{23}$ observed a higher risk of mortality on very cold days in women than men in a case-only study. However, Wolf et al detected similar risks for $\mathrm{MI}$ in association with temperature decreases in both women and men. With regard to body weight, we found temperature effects on fibrinogen only in obese participants with T2D or IGT. Obesity is supposed to be an inflammatory state $^{41}$ and this might influence temperature sensitivity. Nevertheless, Hampel et $a l^{22}$ and Schneider et al ${ }^{20}$ observed no temperature effect modification on fibrinogen by BMI. Regarding PAI-1 and hsCRP, participants with statin intake showed a higher susceptibility to a temperature decrease compared with participants without statin intake. The intake of statins might indicate a poorer overall health, possibly leading to a stronger susceptibility to temperature changes. The temperature-related decrease in hsCRP was only observed in physically active participants with the special genetic background. Since it has been shown that physical activity may reduce inflammation, ${ }^{42}$ we can only speculate that physical activity led to decreases in CRP in association with drops in temperature. 


\section{Strengths and limitations}

One strength of panel studies is the ability to analyse intraindividual variations due to the repeated measurement design. In our study, each participant had up to seven blood withdrawals within a time period of about 11 months. Moreover, each participant serves as his or her own control. Therefore, timeinvariant confounders are accounted for by design, and no control group is needed. We excluded blood samples of participants with acute infection or surgery during the 3 days before the blood withdrawal to exclude the possibility that the observed temperature effects result from an infectious disease. Air pollutants have also been associated with levels of blood markers, ${ }^{43}{ }^{44}$ but it is still unclear whether air pollutants are confounders or effect modifiers of the temperature-blood marker response. However, adjustment for air pollutants hardly changed our results for all blood markers. Therefore, we are confident that the observed temperature effects on blood markers are not confounded by air pollutants. Further strengths are the non-linear confounder adjustment and the information on patient characteristics allowing us to perform subgroup analysis. We only measured outdoor temperature while participants usually spend a lot of time indoors, especially during the cold season. We additionally have to consider a misclassification of personal exposure because we measured the meteorological variables at central measurement sites, even if there is a possible variation of these variables over the study area. However, several studies showed an increase of cardiovascular events in winter, so it is very likely that even a short-term exposure to low outdoor temperatures may influence blood markers. We used a variety of outcome and interaction variables in our analysis; thus, some associations may have occurred by chance. Also, we only looked at a small selection of blood markers that have not or only hardly been studied in association with air temperature. For these reasons, the results can be regarded as first evidence but need to be interpreted with care. In addition, our study population consisted of participants who were either healthy with a special genetic background or who had T2D or IGT. Hence, our results cannot be generalised for the whole population. However, in industrialised countries, T2D is a widespread disease with a growing number of incident cases, and we therefore consider it important to do research on this particularly susceptible population. The limited geographical study area and the restriction to a moderate climate further reduce the generalisability of our results. The detected temperature effects on blood markers are small and might be considered clinically irrelevant. Still, even small changes may indicate a disrupted haemostasis and many acute little changes may have additive effects and lead to a progression of atherosclerosis progression and chronic disease. Moreover, small but consistent associations can give ideas for possible underlying pathways linking temperature and CVD.

\section{CONCLUSIONS}

Our study showed an association between temperature and blood markers of coagulation and inflammation in participants with T2D or IGT and in participants with the special genetic background. The observed differences in temperature effects on blood markers between the two study groups potentially indicate different underlying biologic mechanisms. Our results might partly explain the observed seasonal variation of blood markers and adverse cardiac events.

Acknowledgements We thank Dr Wolfgang Rathmann, DDZ, for performing the glucose tolerance tests and providing us with the $\mathrm{HbA} 1 \mathrm{c}$ measurements. We thank PD Dr Meisinger for coordination of the study in Augsburg, Germany.
Contributors CLS: statistical analyses and interpretation of the results, draft of the manuscript; RH: support in statistical analyses, interpretation of the results and draft of the manuscript, revision of the manuscript after receiving the reviewers' comments; SB: head of data management, support in statistical analyses, interpretation of the results and draft of the manuscript; RR: interpretation of the results and draft of the manuscript; RP: determination of blood marker soluble CD40 Ligand and critical reading of the manuscript; DD-S: determination of blood markers and critical reading of the manuscript; RBD: determination of blood markers $C$ reactive protein, interleukin- 6 and plasminogen activator inhibitor type 1, critical reading of the manuscript; JC: determination of blood markers and critical reading of the manuscript; JS: determination of blood markers and critical reading of the manuscript; RS: determination of blood markers and critical reading of the manuscript: LD: determination of blood markers and critical reading of the manuscript; WK: determination of blood marker fibrinogen and critical reading of the manuscript; JC: determination of the exposure variables and critical reading of the manuscript; UG: data management and critical reading of the manuscript; PB: planning and conduction of the field work and critical reading of the manuscript; UK: planning of the field work and critical reading of the manuscript; AP: conception of the study and critical reading of the manuscript; AES: participation in the conception of the study, interpretation of the results and draft of the manuscript.

Funding This research has been funded wholly or in part by the United States Environmental Protection Agency through STAR (Science to Achieve Results) grant RD 832415 to the University of Rochester. It has not been subjected to the Agency's required peer and policy review and therefore does not necessarily reflect the views of the Agency and no official endorsement should be inferred. This study was supported in part by a grant from the German Federal Ministry of Education and Research (BMBF) to the German Centre for Diabetes Research (DZD e.V.). The KORA research platform (KORA, Cooperative Health Research in the Region of Augsburg) and the MONICA Augsburg studies were initiated and financed by the Helmholtz Zentrum München, German Research Centre for Environmental Health (formerly GSF, National Research Centre for Environment and Health), which is funded by the German Federal Ministry of Education and Research and by the State of Bavaria.

Competing interests None.

Patient consent Obtained.

Ethics approval Ethics approval was provided by Ethics Commission of the Bavarian Chamber of Physicians (Bayerische Landesaerztekammer).

Provenance and peer review Not commissioned; externally peer reviewed.

\section{REFERENCES}

1. Liang WM, Liu WP, Chou SY, et al. Ambient temperature and emergency room admissions for acute coronary syndrome in Taiwan. Int J Biometeorol 2008:52:223-9.

2. Wolf $\mathbf{K}$, Schneider A, Breitner $\mathrm{S}$, et al. Air temperature and the occurrence of myocardial infarction in Augsburg, Germany. Circulation 2009;120:735-42.

3. Iniguez C, Ballester F, Ferrandiz J, et al. Relation between temperature and mortality in thirteen Spanish cities. Int.J Environ Res Public Health 2010;7:3196-210.

4. Braga AL, Zanobetti A, Schwartz J. The time course of weather-related deaths. Epidemiology 2001;12:662-7.

5. Rocklov J, Ebi K, Forsberg B. Mortality related to temperature and persistent extreme temperatures: a study of cause-specific and age-stratified mortality. Occup Environ Med 2011;68:531-6.

6. Rana JS, Arsenault BJ, Despres JP, et al. Inflammatory biomarkers, physical activity, waist circumference, and risk of future coronary heart disease in healthy men and women. Eur Heart J 2011;32:336-44.

7. Koenig W, Khuseyinova N, Baumert J, et al. Increased concentrations of C-reactive protein and IL-6 but not IL-18 are independently associated with incident coronary events in middle-aged men and women: results from the MONICA/KORA Augsburg case-cohort study, 1984-2002. Arterioscler Thromb Vasc Biol 2006;26:2745-51.

8. Ridker PM. C-reactive protein: eighty years from discovery to emergence as a major risk marker for cardiovascular disease. Clin Chem 2009;55:209-15.

9. Sattar N, Murray HM, Welsh P, et al. Are markers of inflammation more strongly associated with risk for fatal than for nonfatal vascular events? PLoS Med 2009;6: e1000099.

10. Rudnicka AR, Rumley A, Lowe GD, et al. Diurnal, seasonal, and blood-processing patterns in levels of circulating fibrinogen, fibrin D-dimer, C-reactive protein, tissue plasminogen activator, and von Willebrand factor in a 45 -year-old population. Circulation 2007;115:996-1003.

11. Elwood PC, Beswick A, O'Brien JR, et al. Temperature and risk factors for ischaemic heart disease in the Caerphilly prospective study. Br Heart J 1993;70:520-3.

12. Woodhouse PR, Khaw KT, Plummer M, et al. Seasonal variations of plasma fibrinogen and factor VII activity in the elderly: winter infections and death from cardiovascular disease. Lancet 1994;343:435-9.

13. Rudez G, Meijer P, Spronk HM, et al. Biological variation in inflammatory and hemostatic markers. J Thromb Haemost 2009;7:1247-55.

14. Sung KC. Seasonal variation of C-reactive protein in apparently healthy Koreans. Int J Cardiol 2006;107:338-42. 
15. Kanikowska D, Sugenoya J, Sato M, et al. Seasonal variation in blood concentrations of interleukin-6, adrenocorticotrophic hormone, metabolites of catecholamine and cortisol in healthy volunteers. Int $\mathrm{J}$ Biometeorol 2009:53:479-85.

16. De Lorenzo F, Kadziola Z, Mukherjee M, et al. Haemodynamic responses and changes of haemostatic risk factors in cold-adapted humans. OJM 1999;92:509-13.

17. Neild PJ, Syndercombe-Court D, Keatinge WR, et al. Cold-induced increases in erythrocyte count, plasma cholesterol and plasma fibrinogen of elderly people without a comparable rise in protein C or factor X. Clin Sci (Lond) 1994;86:43-8.

18. Keatinge WR, Coleshaw SR, Cotter F, et al. Increases in platelet and red cell counts, blood viscosity, and arterial pressure during mild surface cooling: factors in mortality from coronary and cerebral thrombosis in winter. Br Med J (Clin Res Ed) 1984;289:1405-8.

19. Hess KL, Wilson TE, Sauder CL, et al. Aging affects the cardiovascular responses to cold stress in humans. J Appl Physiol 2009;107:1076-82.

20. Schneider A, Panagiotakos D, Picciotto $S$, et al. Air temperature and inflammatory responses in myocardial infarction survivors. Epidemiology 2008;19:391-400.

21. Halonen JI, Zanobetti A, Sparrow D, et al. Associations between outdoor temperature and markers of inflammation: a cohort study. Environ Health 2010;9:42.

22. Hampel R, Breitner $\mathrm{S}$, Ruckerl R, et al. Air temperature and inflammatory and coagulation responses in patients with coronary or pulmonary diseases. Occup Environ Med 2009;67:408-16.

23. Schwartz J. Who is sensitive to extremes of temperature? A case-only analysis. Epidemiology 2005;16:67-72.

24. Mercer JB. Cold-an underrated risk factor for health. Environ Res 2003;92:8-13.

25. Holle R, Happich M, Lowel H, et al. KORA-a research platform for population based health research. Gesundheitswesen 2005:67(Suppl 1):S19-25.

26. Wichmann HE, Gieger C, Illig T. KORA-gen-resource for population genetics, controls and a broad spectrum of disease phenotypes. Gesundheitswesen 2005;67 (Suppl 1):S26-30

27. Hayes JD, Strange RC. Glutathione S-transferase polymorphisms and their biological consequences. Pharmacology 2000;61:154-66.

28. Eiriksdottir G, Smith AV, Aspelund T, et al. The interaction of adiposity with the CRP gene affects CRP levels: age, gene/environment susceptibilty-Reykjavik study. Int.J Obes (Lond) 2009;33:267-72.

29. Jacquemin B, Antoniades C, Nyberg F, et al. Common genetic polymorphisms and haplotypes of fibrinogen alpha, beta, and gamma chains affect fibrinogen levels and the response to proinflammatory stimulation in myocardial infarction survivors: the AIRGENE study. J Am Coll Cardiol 2008;52:941-52.
30. Kolz M, Koenig W, Muller M, et al. DNA variants, plasma levels and variability of $\mathrm{C}$ reactive protein in myocardial infarction survivors: results from the AIRGENE study. Eur Heart J 2008;29:1250-8.

31. Cyrys J, Pitz M, Heinrich J, et al. Spatial and temporal variation of particle number concentration in Augsburg, Germany. Sci Total Environ 2008;401:168-75.

32. Pitz M, Schmid 0, Heinrich J, et al. Seasonal and diurnal variation of PM2.5 apparent particle density in urban air in Augsburg, Germany. Environ Sci Technol 2008; 42:5087-93.

33. Greven S, Küchenhoff H, Peters A. Additive mixed models with P-splines. In: Hinde J, Einbeck J, Newell J, et al, eds. Proceedings of the 21st International Workshop on Statistical Modelling. Galway, Ireland, 3-7 July 2006. 201-207. University of Lancaster, 2006.

34. Grant PJ. Diabetes mellitus as a prothrombotic condition. J Intern Med 2007:262:157-72.

35. Nakaji S, Parodi S, Fontana V, et al. Seasonal changes in mortality rates from main causes of death in Japan (1970-1999). Eur J Epidemiol 2004;19:905-13.

36. Tang JJ, Wang MW, Jia EZ, et al. The common variant in the GSTM1 and GSTT1 genes is related to markers of oxidative stress and inflammation in patients with coronary artery disease: a case-only study. Mol Biol Rep 2010;37:405-10.

37. Duncan BB, Schmidt Ml. The epidemiology of low-grade chronic systemic inflammation and type 2 diabetes. Diabetes Technol Ther 2006;8:7-17.

38. Kafle DR, Shrestha P. Study of fibrinogen in patients with diabetes mellitus. Nepal Med Coll J 2010;12:34-7.

39. Alperovitch A, Lacombe JM, Hanon 0, et al. Relationship between blood pressure and outdoor temperature in a large sample of elderly individuals: the Three-City study. Arch Intern Med 2009;169:75-80.

40. Nayha S, Hassi J, Jousilahti P, et al. Cold-related symptoms among the healthy and sick of the general population: National FINRISK Study data, 2002. Public Health 2011;125:380-8.

41. Gregor MF, Hotamisligil GS. Inflammatory mechanisms in obesity. Annu Rev Immunol 2011;29:415-45.

42. Autenrieth C, Schneider A, Doring A, et al. Association between different domains of physical activity and markers of inflammation. Med Sci Sports Exerc 2009:41:1706-13.

43. Rückerl R, Phipps RP, Schneider A, et al. Ultrafine particles and platelet activation in patients with coronary heart disease-results from a prospective panel study. Part Fibre Toxicol 2007; 4:1

44. Rückerl R, Greven S, Ljungman P, et al. Air pollution and inflammation (interleukin-6 C-reactive protein, fibrinogen) in myocardial infarction survivors. Environ Health Perspect 2007;115:1072-80. 


\section{OEM}

\section{Short-term effects of air temperature on blood markers of coagulation and inflammation in potentially susceptible individuals}

Claudia Luise Schäuble, Regina Hampel, Susanne Breitner, et al.

Occup Environ Med 2012 69: 670-678 originally published online July 25, 2012

doi: 10.1136/oemed-2011-100469

Updated information and services can be found at:

http://oem.bmj.com/content/69/9/670.full.html

These include:

Data Supplement "Supplementary Data"

http://oem.bmj.com/content/suppl/2012/07/24/oemed-2011-100469.DC1.html

References This article cites 43 articles, 11 of which can be accessed free at: http://oem.bmj.com/content/69/9/670.full.html\#ref-list-1

Email alerting

Receive free email alerts when new articles cite this article. Sign up in service the box at the top right corner of the online article.

Notes

To request permissions go to:

http://group.bmj.com/group/rights-licensing/permissions

To order reprints go to:

http://journals.bmj.com/cgi/reprintform

To subscribe to BMJ go to:

http://group.bmj.com/subscribe/ 